\title{
Announcement: best reviewer award 2011
}

\section{Hagit Attiya}

(C) Springer-Verlag 2012

The quality of the journal Distributed Computing depends on a thorough and prompt evaluation procedure. Providing high-quality reviews for submissions, in a timely manner, is essential for any high-quality publication venue.

To recognize the efforts of our reviewers who sustain the evaluation procedure, the editorial board has established a best reviewer award for the journal. The award is designed to recognize those individuals who have consistently demonstrated dedication to the journal. The decision criteria are quality of the reviews, their timeliness and the total number of reviews, over the last three volumes of the journal.
The inaugural award recognizes two of our best reviewers, Danny Hendler, of Ben-Gurion University, Israel, and Dariusz R. Kowalski, of the University of Liverpool, UK.

In addition to meticulous technical reviews, Danny and Darek provided invaluable substantial assistance to authors in improving their manuscripts through detailed suggestions. They are superb examples of outstanding reviewers and deserve our recognition.

Congratulations, Danny and Darek!

Hagit Attiya

Editor-in-chief 